\title{
Erratum to: Tidal decay and stable Roche-lobe overflow of short-period gaseous exoplanets
}

\author{
Brian Jackson $^{1}$ (D) Emily Jensen ${ }^{1}$ - Sarah Peacock ${ }^{2}$. \\ Phil Arras ${ }^{3}$. Kaloyan Penev 4
}

Published online: 9 October 2017

(C) Springer Science+Business Media B.V. 2017

\section{Erratum to: Celest Mech Dyn Astr (2016) 126:227-248 DOI 10.1007/s10569-016-9704-1}

In the original paper, there was an error in Fig. 2. For that figure, we calculated the orbital periods corresponding to the surfaces of young stars for comparison to the short orbital periods of some close-in exoplanets. However, we included an extra factor of $4 \pi^{2}$ in the unit conversion, overestimating the former periods. Figure 2 included here shows the corrected version. Contrary to the erroneous results in the original paper, none of the short-period planets currently orbits interior to the young stellar surface.

In addition, three of the equations contained mistakes. Equation 4 should have read:

$$
\frac{1}{J}\left(\frac{\partial J}{\partial t}\right)_{\dot{\mathrm{M}}_{\mathrm{p}}}=\delta \gamma\left(\frac{\dot{M}_{\mathrm{p}}}{M_{\mathrm{p}}}\right),
$$

i.e., there is no $J$ term on the right-hand side. Equation 9 should have read

$$
\begin{aligned}
\xi\left(\frac{\dot{M}_{\mathrm{p}}}{M_{\mathrm{p}}}\right)+\left(\frac{\partial \ln R_{\mathrm{p}}}{\partial t}\right)_{\mathrm{M}_{\mathrm{p}}} & =\left(\frac{\dot{a}}{a}\right)+\frac{1}{3}\left(\frac{\dot{M}_{\mathrm{p}}}{M_{\mathrm{p}}}\right) \\
& =-2(1-\delta \gamma)\left(\frac{\dot{M}_{\mathrm{p}}}{M_{\mathrm{p}}}\right)+\frac{2}{J}\left(\frac{\partial J}{\partial t}\right)_{\text {tides }}+\frac{1}{3}\left(\frac{\dot{M}_{\mathrm{p}}}{M_{\mathrm{p}}}\right),
\end{aligned}
$$

The online version of the original article can be found under doi:10.1007/s10569-016-9704-1.

\section{Brian Jackson}

bjackson@boisestate.edu

1 Department of Physics, Boise State University, 1910 University Drive, Boise, ID 83725, USA

2 Lunar and Planetary Laboratory, University of Arizona, 1629 E University Blvd, Tucson, AZ 85721-0092, USA

3 Department of Astronomy, University of Virginia, Charlottesville, VA 22904-4325, USA

4 Department of Astrophysical Sciences, Princeton University, Princeton, NJ 08544, USA 
Fig. 2 Orbital periods $P$ versus host star mass $M_{\star}$ for many short-period planets. The lines show the periods corresponding to the stellar surface at 3 (solid, black) and 10 Myrs (dashed, red) after stellar formation as modeled using MESA (Paxton et al. 2011). The data for this plot were harvested from exoplanets.org on 2015 Jul 8

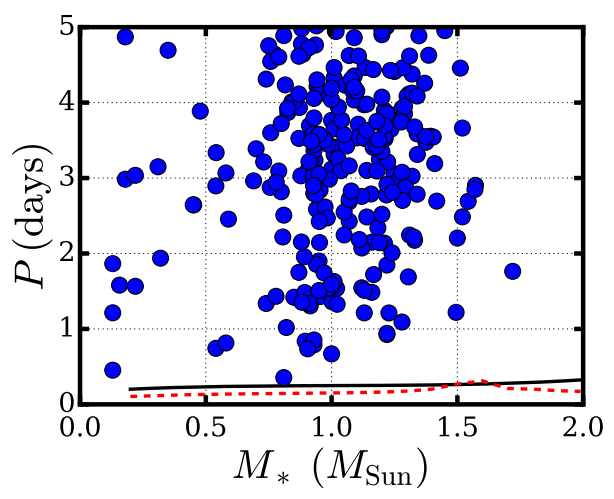

i.e., the rightmost term on the right-hand side was missing. Equation 10 should have read

$$
\left(\frac{\dot{M}_{\mathrm{p}}}{M_{\mathrm{p}}}\right)=-\eta^{-1}\left[\frac{9}{4}\left(\frac{G}{M_{\star}}\right)^{1 / 2} \frac{R_{\star}^{5} M_{\mathrm{p}}}{Q_{\star}} a^{-13 / 2}+\frac{1}{2}\left(\frac{\partial \ln R_{\mathrm{p}}}{\partial t}\right)_{\mathrm{M}_{\mathrm{p}}}\right],
$$

i.e., there should have been a factor of $9 / 4$ in the first term in square brackets and a factor of $1 / 2$ in the second. Fortunately, none of these mistakes affected the model results since the models used the correct relations.

\section{Reference}

Paxton, B., Bildsten, L., Dotter, A., Herwig, F., Lesaffre, P., Timmes, F.: Modules for experiments in stellar astrophysics (MESA). Astrophys. J. Suppl. 192, 3 (2011). doi:10.1088/0067-0049/192/1/3 